\title{
O Uso do Computador como Estratégia Educacional: Relações com a Motivação e Aprendizado de Alunos do Ensino Fundamental
}

\author{
Use of Computers as an Educational Strategy: Relation \\ with Elementary Students' Motivation and Learning
}

\author{
Ibelmar Lluesma Parellada \& Sueli Édi Rufini*, \\ Universidade Estadual de Londrina, Londrina, Paraná, Brasil
}

\begin{abstract}
Resumo
Foram analisadas relações entre uso do computador, motivação e desempenho em prova de conteúdos de matemática em estudantes do ensino fundamental. $\mathrm{O}$ delineamento foi quase experimental, com um grupo experimental e dois de controle. No pré-teste foram avaliados o conhecimento de conteúdos matemáticos e a motivação para vir à escola, por meio da Escala de avaliação da motivação de alunos do ensino fundamental. Na intervenção, o grupo experimental projetou e construiu jogos empregando o computador; o grupo controle 1 fez somente exercícios com lápis e papel (ambos com acesso aos tutoriais); e o grupo de controle 2 assistiu às aulas habituais. Ao final todos os participantes foram avaliados pela segunda vez acerca do conteúdo e da motivação, com os mesmos instrumentos utilizados no pré-teste. Passados 30 dias, os participantes do grupo controle 1 e do grupo experimental fizeram nova prova dos conteúdos de Matemática trabalhados durante a intervenção. Os resultados mostraram que os alunos do grupo experimental tiveram ganhos na qualidade motivacional quando comparados ao grupo de controle 2, indicando que o uso do computador tem importantes implicações para o engajamento e persistência dos alunos em tarefas acadêmicas.

Palavras-chave: Ensino fundamental, motivação, computador, aprendizagem.
\end{abstract}

\begin{abstract}
Relations between the use of computers, motivation and performance of elementary school students in mathematics contents had been analyzed. It was used a quasi-experimental design with an experimental group and two control groups. In the daily pre-test, the knowledge of mathematics contents and the motivation to go to school were evaluated. The experimental group (EG) projected and constructed games using the computer; the control group (CG 1) only worked with pencil and paper (both with access to tutorials); and the control group (CG 2) attended regular classes. In the ending of the study every participant was evaluated for a second time regarding the content and the motivation, with the same instruments used in the daily pre-test. Thirty days later, the participants of CG 1 and EG were once again evaluated regarding mathematics contents which had been worked during the intervention. The results showed that the pupils of the EG were better motivated than the ones of the CG 2, indicating that the use of the computer has important implications for the engagement and persistence of students in academic tasks.

Keywords: Elementary education, theory of self-determination, motivation, computer, learning.
\end{abstract}

A geração atual de alunos do ensino fundamental nasceu na era da informática e não é estranho que muitos deles já dominem a sua linguagem e se relacionem bem com a tecnologia. Segundo Resnick (2007), está disponível atualmente uma grande variedade de produtos que utilizam

"Endereço para correspondência: E-mail: ibelmarp@ gmail.com e sueli_rufini@hotmail.com

Agradecimentos à Coordenação de Aperfeiçoamento de Pessoal de Nível Superior (CAPES), pela bolsa mestrado DS. a tecnologia do computador, recursos tecnológicos de qualidade que parecem facilitar o aprendizado, abrangendo desde softwares a sites educativos.

Um apelo comum para o uso do computador como recurso de ensino é a potencialidade de tornar o aprendizado mais fácil e divertido. No entanto, segundo Papert (1994) os alunos gostam e precisam de desafios para aprender e se desenvolverem, sendo esse um importante argumento para incorporar o computador às atividades escolares. Para Resnick (2007), o uso dos computadores poderia ter impacto positivo no ensino, inclusive na motivação dos 
alunos, pois, com seu intermédio, podem ser realizadas operações complexas, simuladas virtualmente nas telas, sendo também possível interagir com objetos tangíveis, por exemplo, com pequenos robôs ou outros dispositivos eletrônicos. Além disso, os programas podem ser flexíveis, possibilitando a formulação de hipóteses, informando imediatamente acerca dos erros cometidos, os quais podem ser corrigidos passo a passo, por meio de um processo de depuração, além de muitas outras possibilidades.

\section{Motivação no Contexto Escolar}

A motivação humana é um constructo interno, complexo e multideterminado. Diversas teorias elaboradas nos últimos 40 anos colocaram-se diante do desafio de compreendê-la, explicá-la e propor estratégias para intervenção, visando seu incremento. A Teoria da Autodeterminação (TAD), proposta nos anos de 1970, configura-se como uma das principais abordagens desse tema, tendo embasado um grande número de pesquisas em diversas áreas de envolvimento humano: trabalho, saúde, religião, entre outras (Ryan \& Deci, 2002).

A principal premissa da TAD é que os seres humanos têm uma tendência inata para buscar desafios, aprender e dominar novas habilidades. São dotados de uma natureza ativa, voltados para alcançar desenvolvimento, autorregulação e bem-estar. Para que essa tendência inata se desenvolva de forma saudável é imprescindível que sejam atendidas as necessidades psicológicas do ser humano por competência, autonomia e pertencimento. Nesse sentido, o contexto socioambiental tem um papel crucial, podendo nutrir ou prejudicar esses recursos motivacionais internos (Bzuneck \& Guimarães, 2010; Deci \& Ryan, 1985; Ryan \& Deci, 2000).

Especificamente em relação à educação, a TAD permite compreender as interações dos estudantes com seu ambiente escolar e nortear a elaboração de estratégias para promoção da motivação dos estudantes. Segundo Reeve, Deci e Ryan (2004), quando os alunos vêm para a escola trazem consigo um conjunto de valores, interesses e necessidades. O contexto de sala de aula pode oferecer atividades estimulantes, orientações adequadas em relação à aprendizagem e professores com boas estratégias para orientá-los nessa interação. Nesse caso, o contexto educacional pode ser considerado favorável ao desenvolvimento dos estudantes, atuando como um sistema de apoio para nutrir suas necessidades possibilitando, por exemplo, a exploração de interesses, o aprimoramento de habilidades, a internalização de valores e a integração social. Nesse ambiente, é provável que os estudantes apresentem melhor qualidade motivacional, envolvimento, persistência, confiança, entre outras disposições positivas, possibilitando aprendizagem significativa e bom desempenho escolar.

Em contrapartida, caso as interações entre os estudantes e a escola não ocorram de modo adequado, por exemplo, com ênfase excessiva no controle externo, conteúdos compulsórios, prescrições comportamentais rígidas, entre outros, a qualidade da motivação é baixa e dependente de incentivos e ameaças externas. Este tipo de motivação é frágil, sendo associado com baixo envolvimento nas atividades, aprendizagem superficial, propensão a emoções negativas e à evitação de desafios (Reeve et al., 2004).

De acordo com a TAD, a motivação intrínseca é o melhor exemplo de autodeterminação, sendo a atividade em si o foco de interesse e de satisfação, resultando em melhor aprendizagem, criatividade, persistência e outros resultados positivos de desempenho. Por outro lado, há quatro tipos de motivação extrínseca, qualitativamente diferenciados, dependentes do sucesso no processo de internalização de regras e valores externos. A motivação extrínseca por regulação externa e introjetada são os tipos mais controlados de envolvimento, e por regulação identificada e integrada, mais próximos da motivação autodeterminada. O desenvolvimento da qualidade motivacional, previsto no continuum de autodeterminação (desmotivação, motivação extrínseca por regulação externa, introjetada, identificada, integrada e motivação intrínseca) depende das interações com o contexto social.

Os resultados de pesquisas empíricas, indicam que o engajamento dos alunos em tarefas de aprendizagem depende do apoio à sua natureza ativa, o que pode ser alcançado pela criação de contextos favoráveis, com o desenvolvimento e implementação de estratégias de ensino direcionadas a fortalecer suas percepções de competência autonomia e pertencimento (Bzuneck \& Guimarães, 2010; Ryan \& Deci, 2000).

A elaboração de estratégias de ensino, empregando o computador para elaboração ou execução de jogos, potencialmente pode contribuir para a organização de ambientes favorecedores da satisfação das necessidades dos estudantes. Por exemplo, jogando no computador o aluno pode ter a necessidade de competência fortalecida por ser esta uma situação desafiadora, equilibrada com as suas atuais habilidades ou conhecimentos; o senso de autonomia poder ser alcançado, durante o jogo, devido à flexibilidade de escolha dos caminhos para a solução dos problemas de cada etapa, ou de estratégias mais adequadas para a sua conclusão; finalmente, o pertencimento é incrementado pela possibilidade de jogar em rede ou de buscar e oferecer ajuda aos colegas visando ter êxito nos jogos.

\section{O Computador como Recurso de Ensino e Aprendizado: Algumas Evidências Empiricas}

Schacter (1999) reuniu cinco grandes pesquisas, além de duas de menor porte, que vincularam os temas educação e tecnologia procurando destacar os resultados com evidências de implicações educacionais positivas. Em suas conclusões, apresentaram-se como resultados positivos: os alunos que utilizaram instrução baseada no computador registraram, em média, $64 \%$ de acertos em testes, enquanto que os alunos em condições de controle, sem computadores, registraram $50 \%$ de acertos; os estudantes aprenderam mais e em menos tempo quando receberam instrução baseada no computador; os estudantes gostaram mais das aulas e desenvolveram atitudes mais positivas 
quando estas incluíram instrução baseada no computador. Como resultado negativo foi destacado que os computadores não tiveram efeitos positivos em todas as disciplinas para as quais eles foram utilizados.

Ryan, Rigby e Przybylski (2006) conduziram uma pesquisa experimental, com embasamento na Teoria da Autodeterminação, com o intuito de investigar variáveis subjacentes à motivação para os games (jogos no computador). Foram empregados jogos comerciais, além de comunidades que jogavam on-line, sendo analisadas situações em que diversos jogadores estavam conectados em rede. Os resultados mostraram que as necessidades psicológicas básicas de autonomia, competência e pertencimento, independentemente, puderam predizer o prazer no jogo e a intenção de jogar no futuro, além de mudanças no bem-estar antes e depois de jogar, isto é, quando o jogador tinha as necessidades psicológicas básicas satisfeitas havia uma correlação positiva com os itens mencionados acima.

No Brasil, um estudo conduzido por Dwyer et al. (2007) teve como propósito verificar a influência do uso do computador no aprendizado. Foram analisados os resultados do Sistema de Avaliação da Educação Básica (SAEB) de 2001. Nos questionários, específicos para todas as séries e matérias, havia uma pergunta relacionada ao uso de computadores como ferramenta para fazer as lições de casa ou trabalhos da disciplina de Matemática. As respostas dos alunos foram relacionadas com as notas obtidas nas avaliações das disciplinas de Matemática e Português, concluindo-se que o uso do computador não melhora o desempenho do aluno no sistema escolar. Pelo contrário, aqueles que sempre usaram o computador tiveram pior desempenho que outros alunos da mesma classe social.

A pesquisa de Schlünzen e Schlünzen (2006) procurou verificar as relações entre o uso de Tecnologias de Informação e Comunicação (TIC) e o aprendizado de alunos com necessidades especiais. Como resultados, os autores destacaram a superação ou diminuição de barreiras; a inclusão em ambientes sociais e na sala de aula; facilitação do aprendizado; mudança na relação com os familiares, que passaram a observar mais as possibilidades do que as limitações dos alunos; e maior adequação do ensino ao ritmo, possibilidades dos alunos.

Segundo Dwyer et al. (2007), a literatura indica que, apesar das expectativas positivas em relação ao uso do computador na educação, faltam evidências empíricas, pautadas em resultados de estudo experimentais, que sustentem a hipótese de que haja melhoria na aprendizagem e desempenho. No Brasil há relatos de estudos relacionados ao ensino de matemática e física empregando Tecnologias de Informação e Comunicação (TICs). Segundo revisão de Oliveira e Fernandes (2010), apesar de as TICs serem consideradas como elementos didáticos importantes no processo de ensino-aprendizagem de conteúdos matemáticos, são mais relevantes os processos de interação e comunicação entre professores e alunos, assim como as estratégias pedagógicas subjacentes à ação pedagógica.
A questão que norteou a presente pesquisa foi: $\mathrm{O}$ uso do computador como estratégia de ensino promove a motivação e a aprendizagem dos alunos? Buscou-se então descobrir se o emprego do computador para o ensino de conteúdos específicos da disciplina de matemática, na quinta série do ensino fundamental, relaciona-se com a motivação para vir à escola e com o desempenho em prova de conteúdos aprendizagem para um grupo de estudantes.

\section{Método}

A pesquisa foi delineada no modelo quase experimental, adequada aos propósitos do estudo que consistiu em analisar as relações entre uma variável independente, no caso, uma estratégia de ensino e aprendizado com o emprego do computador, e variáveis dependentes, motivação para vir à escola e o desempenho em uma prova de conhecimentos de conteúdos de matemática, de estudantes do ensino fundamental. Todos os procedimentos éticos previstos para a pesquisa envolvendo seres humanos foram observados.

\section{Participantes}

O trabalho foi realizado em uma escola da rede pública estadual de ensino do Estado do Paraná. Participaram da pesquisa 100 alunos das quintas séries, atual sexto ano, com a faixa de idade variando de 10 a 13 anos. É neste nível de escolaridade que são ensinados os conteúdos matemáticos necessários para a elaboração dos jogos selecionados para a intervenção, plano cartesiano e números aleatórios. Entre os alunos que tiveram autorização dos pais para participar, foram indicados 20 pelo professor de Matemática para participarem da etapa de intervenção. O critério adotado para essa indicação foi que o aluno fosse assíduo às aulas, buscando diminuir possíveis ausências de alunos durante a pesquisa.

\section{Instrumentos}

Para avaliar a qualidade da motivação dos alunos foi utilizada a Escala de Avaliação da Motivação de Estudantes do Ensino Fundamental (Rufini, Bzuneck, \& Oliveira, 2011). Após a apresentação de uma questão inicial "Por que você vem à escola?", seguem-se 20 afirmativas, em escala likert de 5 pontos, nas quais o aluno deve assinalar o seu grau de concordância. Cada item corresponde a um tipo ou qualidade de motivação: desmotivação (ausência de intenção para agir), exemplo de item: Não sei, acho que não tem nada para fazer na escola; três tipos de motivação extrínseca, por regulação externa (a atividade é feita para se obter ou escapar de uma consequência externa): Venho à escola para responder a chamada, por regulação introjetada (para evitar sentimentos de culpa ou vergonha): Venho à escola para não deixar meus pais tristes, por regulação identificada (pela identificação pessoal com valor da atividade): Venho à escola porque é aqui que se aprende; e motivação intrínseca (realização da atividade pelo interesse a ela inerente): Venho à escola porque gosto. 


\section{Teste de Conhecimento dos Conteúdos de Matemática}

A prova de conhecimentos incluiu oito questões (quatro objetivas e quatro dissertativas), seis sobre o plano cartesiano, uma sobre números aleatórios e uma sobre variáveis, atribuindo-se o valor de um ponto para cada questão. Antes da aplicação, para verificar a adequação dos itens, o teste foi administrado para um grupo de quatro alunos de quinta série que não participou da pesquisa. A prova foi aplicada nas sessões de pré-teste e pós-teste para grupos experimental e controle 1 .

\section{Tutoriais para Apresentação de Conteúdos}

Foram criadas duas apresentações, baseadas em instrução programada, com a utilização do computador. Tais apresentações constituíram-se de dois tutoriais com desenhos animados, um com os conteúdos de Matemática e o outro com instruções e orientações para elaboração de um jogo no computador. Os tutoriais com a apresentação dos conteúdos de Matemática foram aplicados para o grupo experimental e para o grupo de controle 1. Contudo, apenas o grupo experimental teve acesso ao tutorial para construir jogos no computador e ao programa Scratch, o qual é apropriado para essa finalidade.

\section{O Programa Scratch}

O programa Scratch foi desenvolvido pelo grupo Lifelong Kindergarten do Media Lab no Massachusetts Institute of Technology (MIT), o qual é um dos herdeiros da linguagem de programação LOGO. Está disponível gratuitamente na internet no endereço http://scratch.mit. edu. Comparado a outros programas similares, neste não há como cometer erros de sintaxe nos comandos, podendo o aluno se concentrar na resolução do problema. Além disso, os blocos do programa apenas se encaixam quando a sequência dos comandos for compatível, ou seja, quando faça sentido sintaticamente, pois diferentes tipos de dados possuem diferentes formas, impossibilitando combinações erradas na construção dos comandos.

\section{Procedimentos}

Antes do início da pesquisa, foi realizada uma reunião com os pais de alunos das quintas séries da escola, na qual os pais foram informados a respeito dos objetivos e etapas da pesquisa e assinaram o termo de autorização para participação dos seus filhos.

\section{Pré-Teste}

Os 100 alunos da quinta série da escola, autorizados pelos pais, realizaram o teste de conhecimentos e responderam a Escala de avaliação da motivação. Na sequência, os 20 alunos indicados pelo professor foram divididos, aleatoriamente, em dois grupos de dez alunos cada (5 meninos e 5 meninas), sendo o primeiro denominado grupo experimental e o segundo grupo de controle 1. Para estes dois grupos foram criadas duas apresentações, baseadas em instrução programada, com a utilização do computador.
Tais apresentações constituíram-se de dois tutoriais com desenhos animados, um com os conteúdos de Matemática, números aleatórios e plano cartesiano, e o outro com instruções e orientações para elaboração de um jogo no computador. Os tutoriais com os conteúdos de Matemática foram apresentados tanto para o grupo experimental como para o grupo de controle $1 \mathrm{e}$ apenas o grupo experimental teve acesso ao tutorial para construir jogos no computador e ao programa Scratch, o qual é apropriado para essa finalidade. Para comparar o aprendizado e a motivação controlando o acesso aos conteúdos via computador, optou-se por formar um grupo de controle 2, constituído por 80 alunos das quintas séries que não participaram das atividades usando o equipamento.

\section{Intervenção}

Grupo de Controle 1. As três sessões com o grupo de controle 1 foram organizadas com o objetivo de informar a respeito do trabalho; permitir o acesso ao computador, ligado à internet como uma atividade de recreação; apresentar o tutorial; e resolver os exercícios.

Grupo de Controle 2. O grupo de controle 2 assistiu somente às aulas ministradas pelos seus professores habituais e não recebeu qualquer intervenção; ele serviu para buscar diferença de motivação entre os grupos experimental e de controle 1 que usaram o computador. A avaliação da motivação para este grupo foi feita nas suas salas de aula, no mesmo período de aplicação de pré e pós-teste dos grupos experimental e controle 1 .

Grupo Experimental. Na primeira sessão de intervenção foram apresentados os comandos básicos do programa Scratch, por meio de cinco tutoriais, com a duração total de 12 minutos, baseados em desenhos animados e em instrução programada e de uma apresentação passo a passo envolvendo a execução de determinados comandos. Após assistir a cada módulo do tutorial, o aluno tinha a oportunidade de experimentar e praticar no Scratch o que havia visto. Na segunda sessão, houve a elaboração de um jogo utilizando-se os conteúdos apresentados nos tutoriais. A proposta do jogo consistia em fazer dois personagens se encontrarem em um determinado ponto da tela, utilizando-se, para isto, dois controles, um para o eixo x e o outro para o eixo y. O objetivo era colocar os números corretos para as posições nos eixos $\mathrm{x}$ e $\mathrm{y}$ para que o desenho " $A$ " pudesse encontrar o desenho "B". Este último, tinha sua posição nos eixos x e y sorteadas anteriormente, o que correspondia à coordenada do desenho.

Os alunos foram incentivados a personalizar seu jogo escolhendo seus próprios desenhos e a utilizar comandos diferenciados, se desejassem.

Pós-Teste. Nesta sessão, para os grupos experimental e controle 1, por meio de tutoriais, foram apresentados o plano cartesiano, números aleatórios e variáveis, assegurando-se semelhança na informação recebida por ambos. Na sequência, foi entregue uma folha de exercícios, uma 
Parellada, I. L. \& Rufini, S. E. (2013). O Uso do Computador como Estratégia Educacional: Relações com a Motivação e Aprendizado de Alunos do Ensino Fundamental.

atividade de aprendizagem, sendo opcional para o grupo experimental o uso do programa Scratch ou de lápis e papel para resolver o item número 8. Para o grupo de controle 1 havia a obrigatoriedade de fazer a atividade somente com o lápis e papel.

O oitavo item da folha de exercícios solicitava a criação de um jogo, que poderia ser uma corrida de barcos, de foguetes, de carros, ou qualquer outro desenho que o aluno desejasse utilizar. $\mathrm{O}$ jogo deveria ser construído pelo aluno com base nos conteúdos de matemática que ele havia aprendido nas sessões anteriores. Os estudantes de ambos os grupos estavam livres para consultar os tutoriais vistos anteriormente, solicitar ajuda; comunicarem-se e auxiliarem-se mutuamente. Ao final da sessão os dois grupos responderam a avaliação da qualidade motivacional.

Reteste. Passados 30 dias da última sessão foi reaplicado para o grupo controle 1 e experimental o teste de conhecimento dos conteúdos utilizado nas sessões pré e pós-teste, apenas com modificações nos valores das coordenadas. O objetivo foi verificar se haveria diferença entre os grupos na recordação dos conteúdos trabalhados o plano cartesiano, números aleatórios e variáveis.

\section{Resultados}

\section{Teste de Conhecimentos}

Em razão da distribuição dos desempenhos nas avaliações realizadas não se ajustarem à distribuição normal além do pequeno número de participantes, foi utilizada estatística não paramétrica. O teste de Mann Whitney aplicado aos resultados do pré-teste de conhecimento dos grupos experimental e de controle $1, p=0,272$, indicou que não houve diferenças significativas, os alunos dos dois grupos sabiam pouco acerca dos conteúdos propostos, ou seja, partiram de um mesmo nível de conhecimentos.

O teste Wilcoxon, aplicado para análise dos resultados do pré e pós-teste indicou diferenças significativas no número de acertos, tanto para os estudantes do grupo experimental, $p$-valor (bilateral) $=0,010$, quanto para os do grupo de controle $1, p$-valor (bilateral) $=0,007$, isto é, os dois grupos apresentaram uma maior pontuação no pós-teste. O teste de Mann Whitney revelou que não houve diferença significativa entre o desempenho do grupo de controle 1 e o grupo experimental no pós-teste, significando não ter havido diferença de aprendizado dos conteúdos entre os dois grupos, $p=0,469$. De acordo com os testes de Wilcoxon e Mann Whitney não houve diferença entre o grupo experimental e o grupo de controle 1 no teste de consistência do aprendizado, realizado 30 dias após o término da última sessão.

\section{Avaliação da Motivação para Vir à Escola}

Buscando-se comparar a motivação dos participantes do grupo experimental, de controle 1 , e controle 2 na etapa de pré-teste, foi aplicado o teste de Kruskal-Wallis o qual não revelou diferenças significativas entre os três grupos (Tabela 1).

Tabela 1

Estatísticas de Média, Desvio Padrão e Mediana dos Grupos de Estudo no Pré-Teste, e Valor de p do Teste de Kruskal-Wallis

\begin{tabular}{|c|c|c|c|c|c|c|c|}
\hline \multirow{2}{*}{$\begin{array}{l}\text { Variáveis } \\
\text { Desmotivação }\end{array}$} & \multicolumn{2}{|c|}{$\begin{array}{l}\text { Experimental } \\
\quad(n=10)\end{array}$} & \multicolumn{2}{|c|}{$\begin{array}{c}\text { Controle } 1 \\
\quad(n=10)\end{array}$} & \multicolumn{2}{|c|}{$\begin{array}{c}\text { Controle } 2 \\
(n=80)\end{array}$} & \multirow{2}{*}{$\begin{array}{l}p^{*} \\
0,32\end{array}$} \\
\hline & $5,6 \pm 2,37$ & $5,0 \mathrm{a}$ & $5,4 \pm 4,22$ & $3,0 \mathrm{a}$ & $4,8 \pm 2,87$ & $3,0 \mathrm{a}$ & \\
\hline Externa & $4,6 \pm 2,07$ & $3,5 \mathrm{a}$ & $6,2 \pm 3,08$ & $5,5 \mathrm{a}$ & $5,76 \pm 3,32$ & $5,0 \mathrm{a}$ & 0,48 \\
\hline Introjetada & $8,0 \pm 3,56$ & $7,0 \mathrm{a}$ & $8,4 \pm 4,33$ & $7,0 \mathrm{a}$ & $8,51 \pm 3,98$ & $8,0 \mathrm{a}$ & 0,93 \\
\hline Identificada & $13,2 \pm 2,90$ & $15,0 \mathrm{a}$ & $14,3 \pm 1,34$ & $15,0 \mathrm{a}$ & $14,18 \pm 2,34$ & $15,0 \mathrm{a}$ & 0,55 \\
\hline Motivação intrínseca & $9,5 \pm 4,11$ & $10,5 \mathrm{a}$ & $11,6 \pm 4,17$ & $13,5 \mathrm{a}$ & $11,16 \pm 3,38$ & $11,0 \mathrm{a}$ & 0,3 \\
\hline
\end{tabular}

Nota. Medianas seguidas por letras diferentes indica diferença estatisticamente significativa pelo teste de Dunn ao nível de significância de $5 \%$.

Comparando-se o desempenho dos participantes do grupo experimental, nas etapas pré e pós-teste, de acordo com os resultados do teste Wilcoxon, descobriu-se diferença significativa em relação à desmotivação, $p$-valor (bilateral $)=0,016$, diminuindo de 5,0 pontos para 3,0 pontos. Além disso, houve uma diferença significativa da motivação extrínseca por regulação introjetada, $p$-valor (bilateral) $=0,029$, passando de uma pontuação média de 7,0 para 3,0 pontos (Tabela 2). Não foram verificadas diferenças no desempenho dos participantes do grupo de controle 1 nas etapas de pré e pós-teste. 
Psicologia: Reflexão e Crítica, 26(4), 743-751.

Tabela 2

Resultado do Teste Wilcoxon para a Diferença entre o Pré e Pós-Teste, Grupo Experimental

\begin{tabular}{|c|c|c|c|c|c|c|c|c|c|}
\hline \multirow{2}{*}{ Variáveis } & \multicolumn{4}{|c|}{ Pré-teste } & \multicolumn{4}{|c|}{ Pós-teste } & \multirow{2}{*}{$p$} \\
\hline & $N$ & Med. & M. A. & $D P$ & $N$ & Med. & M.A. & $D P$ & \\
\hline Desmotivação & 10 & 5 & 5,6 & 2,36 & 10 & 3 & 3 & 0,0 & 0,01 \\
\hline Externa & 10 & 3,5 & 4,6 & 2,06 & 10 & 3 & 3,7 & 1,05 & 0,18 \\
\hline Introjetada & 10 & 7 & 8 & 3,55 & 10 & 3 & 4,5 & 3,71 & 0,02 \\
\hline Identificada & 10 & 15 & 13,2 & 2,89 & 10 & 14 & 13 & 2,58 & 0,88 \\
\hline Intrínseca & 10 & 10,5 & 9,5 & 4,11 & 10 & 11 & 10,1 & 4,01 & 0,84 \\
\hline
\end{tabular}

Comparando-se os resultados do pré-teste e o pós-teste, obtidos pelo grupo de controle 2 (grupo que não participou da intervenção), descobriu-se diferença significativa na motivação extrínseca por regulação externa, $p$-valor (bila- teral $)=0,004$, isto é, os alunos perceberam-se controlados externamente, conforme Tabela 3.

$\mathrm{Na}$ Tabela 4 estão apresentados os resultados do teste Kruskal-Wallis para avaliar as diferenças entre os grupos experimental, controle 1 e controle 2 no pós-teste.

Tabela 3

Resultados do Teste de Wilcoxon para a Diferença entre o Pré-Teste e o Pós-Teste do Grupo de Controle 2

\begin{tabular}{lcccccccccc}
\hline \multirow{2}{*}{ Variáveis } & \multicolumn{9}{c}{ Pré-teste } & \multicolumn{5}{c}{ Pós-teste } & $p$ \\
\cline { 2 - 7 } & $N$ & Med. & M. A. & $D P$ & $N$ & Med. & M.A. & $D P$ & \\
\hline Desmotivação & 80 & 3,0 & 4,80 & 2,87 & 80 & 3,5 & 5,16 & 3,24 & 0,38 \\
Externa & 80 & 5,0 & 5,76 & 3,31 & 80 & 6,0 & 6,70 & 3,75 & 0,004 \\
Introjetada & 80 & 8,0 & 8,51 & 3,98 & 80 & 8,0 & 8,18 & 3,61 & 0,493 \\
Identificada & 80 & 15,0 & 14,17 & 2,34 & 80 & 15,0 & 13,75 & 2,60 & 0,091 \\
Intrínseca & 80 & 11,0 & 11,16 & 3,38 & 80 & 11,0 & 10,70 & 3,70 & 0,279 \\
\hline
\end{tabular}

Tabela 4

Estatísticas de Média, Desvio Padrão, Mediana dos Grupos de Estudo no Pós-Teste, e o Valor de p do Teste de Kruskal-Wallis

\begin{tabular}{|c|c|c|c|c|c|c|c|}
\hline \multirow{2}{*}{$\begin{array}{l}\text { Variáveis } \\
\text { Desmotivação }\end{array}$} & \multicolumn{2}{|c|}{$\begin{array}{c}\text { Experimental } \\
\quad(n=10)\end{array}$} & \multicolumn{2}{|c|}{$\begin{array}{c}\text { Controle } 1 \\
\quad(n=10)\end{array}$} & \multicolumn{2}{|c|}{$\begin{array}{c}\text { Controle } 2 \\
(n=80)\end{array}$} & \multirow{2}{*}{$\begin{array}{c}p \\
0,0005\end{array}$} \\
\hline & $3,0 \pm 0,00$ & $3,0 \mathrm{a}$ & $3,0 \pm 0,00$ & $3,0 \mathrm{a}$ & $5,2 \pm 3,25$ & $3,5 b$ & \\
\hline Externa & $3,7 \pm 1,06$ & $3,0 \mathrm{a}$ & $5,4 \pm 2,84$ & $4,0 \mathrm{ab}$ & $6,7 \pm 3,76$ & $6,0 \mathrm{~b}$ & 0,0351 \\
\hline Introjetada & $4,5 \pm 3,72$ & $3,0 \mathrm{a}$ & $6,0 \pm 3,50$ & $5,0 \mathrm{ab}$ & $8,19 \pm 3,62$ & $8,0 \mathrm{~b}$ & 0,0011 \\
\hline Identificada & $13,0 \pm 2,58$ & $14,0 \mathrm{a}$ & $13,5 \pm 2,12$ & $14,0 \mathrm{a}$ & $13,75 \pm 2,61$ & $15,0 \mathrm{a}$ & 0,0864 \\
\hline Motivação intrínseca & $10,1 \pm 4,01$ & $11,0 \mathrm{a}$ & $11,2 \pm 3,74$ & $12,0 \mathrm{a}$ & $10,7 \pm 3,71$ & $11,0 \mathrm{a}$ & 0,7735 \\
\hline
\end{tabular}


Parellada, I. L. \& Rufini, S. E. (2013). O Uso do Computador como Estratégia Educacional: Relações com a Motivação e Aprendizado de Alunos do Ensino Fundamental.

Observe-se que o grupo experimental e o controle 1 diferiram do controle 2 na desmotivação $(p<0,001)$, e na motivação extrínseca por regulação externa $(p=0,0351)$. Em relação à motivação extrínseca por regulação introjetada $(p=0,001)$ o grupo experimental diferiu apenas do controle $2 \mathrm{Na}$ análise da motivação extrínseca por regulação identificada $(p=0,0864)$ e da motivação intrínseca $(p=0,774)$ não foram detectadas diferenças significativas entre os grupos experimental, controle 1 e controle 2 . $\mathrm{Na}$ análise de todos os tipos de motivação observou-se que o grupo experimental não diferiu significativamente do grupo controle $1(p>0,05)$.

\section{Discussão}

Neste estudo, buscou-se descobrir se uma estratégia de ensino e aprendizado, empregando o computador como recurso, relaciona-se com a motivação e aprendizagem de um grupo de estudantes do ensino fundamental. Os participantes partiram de uma base de conhecimento semelhante e obtiveram resultados similares no pós-teste e reteste. De certo modo, este não foi um resultado surpreendente, pois as condições objetivas deste estudo não permitiam uma intervenção longitudinal, como, por exemplo, a pesquisa realizada por Harel (1991), na qual crianças da quarta série construíram programas para ensinar frações para outros estudantes e aprenderam esse conceito matemático melhor do que alunos que utilizaram métodos convencionais de aprendizado. Talvez fosse necessário mais tempo de trabalho para que ocorresse um aprendizado em profundidade. Contudo, a criação de um ambiente diferenciado de ensino e aprendizagem, no qual a motivação dos alunos pudesse ser verificada, trouxe importantes implicações educacionais.

A qualidade motivacional dos participantes era semelhante quando a pesquisa foi iniciada. No término da pesquisa, o grupo de controle 2, o qual não participou da intervenção e assistiu apenas às aulas habituais, apresentou desempenho superior na avaliação da desmotivação e dos tipos controlados de motivação extrínseca, comparado com os grupos experimental e de controle 1. Há evidências na literatura (Bzuneck \& Guimarães, 2010; Deci \& Ryan, 2000; Reeve et al., 2004; Reis, Sheldon, Gable, Roscoe, \& Ryan 2000) de que no contexto de aprendizagem escolar a motivação autônoma relaciona-se com resultados positivos no desenvolvimento de habilidades, criatividade, persistência, processamento profundo de informações, bem-estar emocional, entre outros. Em contraposição, um aluno desmotivado não tem intenção para agir porque desvaloriza ou não encontra, naquela situação de realização, elementos que despertem seu interesse. No caso da motivação controlada, a intenção é o desdobramento de coerção externa ou intrapsíquica (motivação extrínseca por regulação externa e introjetada), sendo vinculada a baixos resultados de desempenho e aprendizagem.

A diferença da motivação encontrada entre os grupos pode ser relacionada ao uso do computador, tendo em vista o seu poder de atração e de interesse para os estudantes, levando-os a valorizarem e se identificarem com as atividades propostas. Mesmo para o grupo de controle 1, que não pode usar o software para a realização da atividade, o uso dos tutorias pode ter despertado um interesse, ainda que situacional, pelo conteúdo apresentado. Nesse caso, entre o grupo experimental e o controle 1, na sessão pós-teste, não foram registradas diferenças, provavelmente porque os alunos do grupo de controle 1 participaram de atividades que incluíram o uso de computadores para navegar na Internet em sites que eles podiam escolher. Essas atividades foram propostas para procurar diminuir a possibilidade de faltas, já que os estudantes desse grupo participariam apenas de testes de conhecimento dos conteúdos de Matemática e da avaliação da motivação. Além disso, apesar de não utilizarem o software para realizar a atividade de aprendizagem, os alunos do grupo de controle 1 também assistiram à apresentação dos conteúdos de Matemática pelo computador, em forma de desenhos animados. Entretanto, este não seria o procedimento usual em uma sala de aula tradicional e pode ter influenciado positivamente a motivação do grupo de controle 1 .

Ainda que a diferença entre o grupo experimental o de controle 1 e não tenha sido significativa, é interessante observar que o valor da mediana do grupo de controle 1 nas avaliações da motivação por regulação externa e introjetada foi maior do que a do grupo experimental. Foi possível observar que as pontuações relativas à avaliação da motivação extrínseca por regulação identificada e motivação intrínseca, foram sempre altas em todos os grupos, um dado positivo, indicando que, de modo geral, os alunos gostam de vir para a escola.

Comparados os resultados de avaliação da motivação de cada grupo no pré-teste e pós-teste, o grupo experimental apresentou diminuição na desmotivação e na motivação extrínseca por regulação introjetada. Por outro lado, o grupo de controle 1 não sofreu mudanças em sua qualidade motivacional e o grupo de controle 2 aumentou seu desempenho na avaliação da motivação extrínseca por regulação externa. Neste caso, a intervenção parece ter se relacionado com a motivação, pois para os alunos a quem foi dada a opção do uso do programa Scratch, recurso este potencialmente promotor de autonomia, houve diminuição da motivação controlada.

Uma das hipóteses do presente estudo era que a construção de um jogo como atividade de aprendizagem, usando o computador como ferramenta, promoveria a liberdade de escolhas e nutriria a necessidade psicológica básica de autonomia. A possibilidade de escolhas pessoais é uma das condições necessárias para a percepção de que comportamento está emanando do self, isto é, o aluno se percebe origem de seu próprio comportamento (Deci \& Ryan, 2000). Neste sentido, o contexto de aprendizado deve facilitar o engajamento com a utilização de recursos de ensino que possibilitem escolhas pessoais e que se vinculem às preferências dos alunos. Outros fatores relacionados à autonomia foram pesquisados por Lepper 
e Cordova (1996), os quais apontaram para a importância da contextualização, personalização e da possibilidade de escolhas.

A percepção da necessidade psicológica básica de competência também é uma questão importante para o aprendizado (Reeve et al., 2004). Ao utilizar o computador para a construção dos jogos, cada estudante pôde testar suas hipóteses passo a passo. Nesse caso, o professor constituiu-se num mediador, que orientava o aluno apenas quando necessário. Em tal situação de ensino e aprendizagem, o professor tem a oportunidade de acompanhar as dúvidas do aluno, pois elas são explicitadas por meio de objetos tangíveis, o que facilita localizar as eventuais dificuldades e, quando necessário, ajustar o grau de dificuldade de uma atividade. $\mathrm{O}$ grau de dificuldade de uma atividade está relacionado com o conhecimento prévio do aluno; por isso, o professor deve conhecer muito bem os seus alunos e criar um ambiente que possibilite desafios compatíveis com as condições atuais do aluno. A necessidade psicológica básica de competência depende da percepção que o aluno tem de que ele está interagindo de forma eficiente com o seu meio. Caso o grau de dificuldade esteja acima de suas possibilidades, sua necessidade de competência será frustrada, acarretando prejuízos para o desenvolvimento de seu aprendizado (Ryan \& Deci, 2000).

Finalmente, a estratégia de ensino empregada pode ter incentivado e fortalecido o vínculo entre os alunos, pois eles se reuniam e discutiam entre si, devido ao fato de serem possíveis configurações diferentes para os jogos. Era comum experimentarem diversos desenhos e sons e diferentes comandos, antes de concluírem a atividade, o que possibilitou aproximação do grupo e favoreceu o surgimento de vínculos afetivos, nutrindo a necessidade de pertencimento dos alunos, fato que, em última instância, favoreceu a qualidade da motivação do grupo de alunos para vir à escola.

\section{Considerações Finais}

Uma estratégia de ensino que contribua para evitar que os estudantes fiquem desmotivados ou apresentem baixa qualidade motivacional tem importantes implicações acadêmicas, pois, de acordo com Ryan e Deci (2000), a qualidade da motivação tem influência direta na qualidade do aprendizado. Um aluno autodeterminado persiste em suas atividades educacionais, mobilizando-se no sentido de assumir responsabilidade pelos seus estudos. Este comportamento contribui para um aprendizado em profundidade, melhor desempenho acadêmico, bem-estar, e melhor inserção no contexto social. A motivação do estudante está ligada intimamente com o contexto educacional, o qual tem influência direta na qualidade de seu aprendizado. Sendo assim, quando o aprendiz tem a percepção de que o aprendizado é significativo, ele se engaja de forma ativa no processo (Reeve et al., 2004).

Para favorecer o surgimento da motivação autônoma são necessários, além de uma estratégia adequada, meios objetivos para apoiá-la. Isso não significa apenas equipar as instituições educacionais com computadores, entre outros equipamentos; é necessário formar o professor para o uso adequado daquelas ferramentas. Isso demanda um tipo de formação de professores que não é muito frequente em nosso país, como indicado no estudo de Hummel (2007), que era usual os professores investigados empregarem o computador como um caderno digital, enfrentando dificuldades para desenvolver seu trabalho, particularmente com os conteúdos referentes à Matemática.

Papert (1999) faz uma analogia para explicar a ineficiência de introduzir novos recursos na escola sem compreender o que eles significam. $\mathrm{O}$ autor imagina um cenário no qual houvesse escolas, mas ainda não houvesse a escrita, inexistindo, portanto, a tecnologia do livro e nem a do lápis. O conhecimento seria transmitindo verbalmente. Um dia é inventado o lápis e a escrita, e alguém imagina que o lápis poderia ser de grande valia para a educação e o introduz na sala de aula, esperando que assim o aprendizado fosse revolucionado, podendo surgir ideias que defendessem a disponibilização de um lápis em cada sala de aula do país. Certamente, essas medidas mudariam pouco o sistema educacional, pois, a essência do aprendizado não está no lápis, ela se encontra no uso que fazemos dele. Outra questão levantada por Papert (1994) é que os recursos devem estar à disposição do aluno sempre que for necessário, o que não ocorre na prática, pois os computadores nem sempre estão acessíveis.

Nutrir as necessidades psicológicas básicas dos alunos, colocando à sua disposição os meios adequados para a expressão de escolhas pessoais significativas, personalização das atividades e cooperação, além de desafios adequados, evitando-se um clima de competição, tem influência na motivação dos alunos, podendo afetar a qualidade do aprendizado, o engajamento e a persistência dos estudantes nas tarefas acadêmicas. Certamente, mais pesquisas são necessárias para a consolidação desses dados; contudo, a motivação é um fator de fundamental importância para o futuro acadêmico do aluno, para a aprendizagem e desenvolvimento saudável.

\section{Referências}

Bzuneck, J. A., \& Guimarães, S. É. R (2010). A promoção da autonomia como estratégia motivacional. In E. Boruchovitch, J. A. Bzuneck, \& S. É. R. Guimarães (Eds.), Motivação para aprender (pp. 13-70). Petrópolis, RJ: Vozes.

Deci, E. L., \& Ryan, R. M. (1985). Intrinsic motivation and selfdetermination in human behavior. New York: Plennum Press.

Deci, E. L., \& Ryan, R. M. (2000). The "what" and "why" of goal pursuits: Human needs and self-determination of behavior. Psychological Inquiry, 11(4), 227-268.

Dwyer, T., Wainer, J., Dutra, R. S., Covic, A., Magalhães, V. B., Ferreira, L. R. R., \& Pimenta, V. A. (2007). Desvendando mitos: Os computadores e o desempenho no sistema escolar. Educação \& Sociedade, 28(101), 1303-1328.

Harel, I. (1991). Children designers: Interdisciplinary constructions for learning and knowing mathematics in a computerrich school. Westport, CT: Ablex. 
Parellada, I. L. \& Rufini, S. E. (2013). O Uso do Computador como Estratégia Educacional: Relações com a Motivação e Aprendizado de Alunos do Ensino Fundamental.

Hummel, E. I. (2007). A formação de professores para o uso da informática no processo de ensino e aprendizagem de alunos com necessidades educacionais especiais em classe comum (Dissertação de mestrado em Educação, Universidade Estadual de Londrina, Londrina, PR, Brasil).

Lepper, M. R., \& Cordova, D. I. (1996). Intrinsic motivation and the process of learning: Beneficial effects of contextualization, personalization, and choice. Journal of Educational Psychology (Washington), 88(4), 715-730.

Oliveira, G. P., \& Fernandes, R. U. (2010). O uso de tecnologias para ensino de trigonometria: Estratégias pedagógicas para a construção significativa da aprendizagem. Educação Matemática, 12(3), 548-577.

Papert, S. (1994). A máquina das crianças. Porto Alegre, RS: Artes Médicas.

Papert, S. (1999). Diversity in learning: A vision for the new Millennium [DVD]. Dallas, TX: Diversity Task Force. Retrieved August 23, 2008, from http://www.papert.org/articles/ diversity/DiversityinLearningPart1.html

Reeve, J., Deci, E. L., \& Ryan, R. M. (2004). Self-determination theory: A dialetical framework for understanding socio cultural influences on student motivation. In D. M. Mcinerney \& S. Van Etten (Eds.), Big theories revisited (pp. 31-60). Greenwich, UK: Information Age.

Reis, H. T., Sheldon, K., Gable, S. L., Roscoe, J., \& Ryan, R. M. (2000). Daily well-being: The role of autonomy, competence, and relatedness. Personality and Social Psychology Bulletin, 26, 419-435.

Resnick, M. (2007). Sowing the seeds for a more creative society. International society for technology in education. Retrieved May 12, 2007, from http://web.media.mit.edu/ mres/papers. html

Rufini, S. É., Bzuneck, J. A., \& Oliveira, K. L. (2011). Estudo de validação de uma medida de avaliação da motivação para alunos do ensino fundamental. Psico-USF, 16, 1-9.

Ryan, R. M., \& Deci, E. L. (2000). Self-Determination Theory and the facilitation of intrinsic motivation, social development, and well-being. The American Psychologist, 55, 68-78

Ryan, R. M., \& Deci, E. L. (2002). An overview of self-determination theory. In E. L. Deci \& R. M. Ryan (Eds.), Handbook of self-determination research (pp. 3-33). Rochester, NY: University of Rochester Press.

Ryan, R. M., Rigby, C. S., \& Przybylski, A. (2006). Motivation pull of video games: A self-determination theory approach. Motivation and Emotion, 30(4), 347-365.

Schacter, J. (1999). The impact of education technology on student achievement: What the most current research has to say. Santa Monica, CA: Milken Exchange on Education Technology.

Schlünzen, E. T. M., \& Schlünzen, J. K., Jr. (2006). Tecnologias, desenvolvimento de projetos e inclusão de pessoas com deficiência. Inclusão: Revista da Educação Especial, 2, 46-51. 